\title{
Déterminisme génétique de dix systèmes enzymatiques chez Hedysarum coronarium L.
}

\author{
N. Trifi-Farah ${ }^{1}$, W.-S. Chatti ${ }^{2}$, M. Marrakchi ${ }^{2}$ et J. Pernès ${ }^{3}$ \\ 1 Ecole Nationale des Ingénieurs B.P.W. 3038 Sfax, Tunisie; \\ 2 Laboratoire de Génétique. Faculté des Sciences de Tunis Campus Universitaire 1060 Tunis, Tunisie; \\ ${ }^{3}$ Laboratoire de Génétique et Physiologie du Développement des Plantes. C.N.R.S. 91190 Gif-Sur-Yvette, France
}

(reçu le 4 mai 1988, accepté le 3 mars 1989)

Résumé - Dix systèmes enzymatiques ont été utilisés pour évaluer le polymorphisme enzymatique chez quelques populations d'Hedysarum coronarium. Il s'agit de : alcool déshydrogénase (ADH), malate déshydrogénase (MDH), isocitrate déshydrogénase (ICD), phosphogluconate déshydrogénase (6-PGD), peroxydase (PER), glutamate oxaloacétate transaminase (GOT), phosphoglucomutase (PGM), estérases (EST), phosphatases acides (PHO) et phosphoglucoisomérase (PGI).

L'analyse des résultats obtenus a permis d'émettre des hypothèses relatives à la structure des enzymes et à leur déterminisme génétique, certaines de ces hypothèses ont été vérifiées grâce à la ségrégation des bandes isoenzymatiques dans la descendance de deux familles issues d'autofécondation (GOT, 6-PGD et PGI). Les proportions mendéliennes sont respectées pour ces 3 enzymes. L'ensemble de ces données sera exploité pour l'étude du polymorphisme des populations de cette espèce.

Hedysarum coronarium - isozymes - structure - contrôle

Summary - Genetic determinism of ten enzymatic systems in Hedysarum coronarium L. Ten enzyme systems were used in the study of the enzymatic polymorphism of some populations of Hedysarum coronarium : alcohol dehydrogenase $(A D H)$, malate dehydrogenase $(\mathrm{MDH})$, isocitrate dehydrogenase (ICD), phosphogluconate dehydrogenase (6-PGD), peroxydase (PER), glutamate oxaloacetate transaminase (GOT), phosphoglucomutase (PGM) esterases (EST), acid phosphatases (PHO) and phosphogluco-isomerase (PGI). These results suggest some hypotheses concerning the enzyme structure and the genetic determinism of these enzymes. These hypotheses were confirmed for three enzymatic systems : GOT, 6-PGD and PGI, using isozyme bands segregation for two selfed progenies. Mendelian segregations were observed for these three enzymes.

The obtained results will be used in the study of population's polymorphism in this species.

H. coronarium - isozymes - structure - genetic control

\section{Introduction}

Hedysarum coronarium L. (Légumineuse, papilionacée) est une plante diploïde $(2 n=16)$ préférentiellement allogame avec un faible degré d'autogamie estimé à 10\% (Chriki et al., 1984).

Cette espèce, appelée couramment sainfoin d'Espagne ou sulla, pousse spontanément sur des sols variés dont elle permet la protection contre l'érosion et l'enrichissement en azote assimilable (Baatout et al., 1976).

En Tunisie, on la rencontre dans le nord où elle présente des peuplements dans le Tell à par- tir de $300 \mathrm{~mm}$ de pluies et jusqu'à $1000 \mathrm{~m}$ d'altitude (Figier, 1982).

Sur le plan agronomique, le sulla présente un intérêt important en particulier pour la production de fourrage. En effet, dans les stations d'origine, ces plantes, qui poussent spontanément, sont exploitées par les agriculteurs, aussi bien pour la pâture que pour la protection des sols, surtout dans les pentes marneuses. Sa culture, bien que limitée, permet la production d'importantes quantités de fourrage pouvant atteindre jusqu'à 90 tonnes de poids frais par hectare (Le Houerou, 1965), et dont la qualité est comparable à celle 
de la luzerne et au trèfle violet (Maymone et al., 1951; Cenni et al., 1968; Ballatore, 1972).

Plusieurs travaux effectués sur les populations naturelles de $H$. coronarium, prospectées essentiellement en Tunsie, ont montré l'existence d'une variabilité morphologique importante (Combes et al., 1975; Figier et al., 1977; Chriki, 1986; Chatti, 1987). Dans le but de mieux analyser cette variabilité, nous avons entrepris l'étude du polymorphisme électrophorétique de dix systèmes enzymatiques. Le déterminisme génétique de ces enzymes doit être analysé pour permettre l'étude précise en terme de fréquences génotypiques et alléliques de la structure des populations. Nous présentons ici les systèmes choisis et l'analyse génétique des descendances en ségrégation. L'utilisation de ces systèmes a pour but de décrire le polymorphisme des populations de cette espèce.

\section{Matériel et Méthodes}

\section{Matériel végétal}

Le matériel végétal utilisé dans ce travail comprend:

- Cinq populations spontanées d'origine tunisienne (Fig. 1). Il s'agit de Béjà (BES), El-Haouaria (EHS), Djebel Zit (ZTS), Forêt Aîn Djamala (FAS) et Tunis (TUS);

- Cinq cultivars semés en Tunisie dans les régions de Béjà (BEC), Testour (TEC), Nefza (NEC), Chaouat (CHC) et Mateur (MAC). Ces cultivars appartiennent à des introductions italiennes de semences mal définies et appartenant au moins aux variétés Scaravatti, Sparacia et Grimaldi;

- Deux populations "synthétiques" appelées ZE1 et EZ1 obtenues de la façon suivante : un individu de la population ZTS caractérisé par un port prostré marqué par la présence d'importantes ramifications plagio-

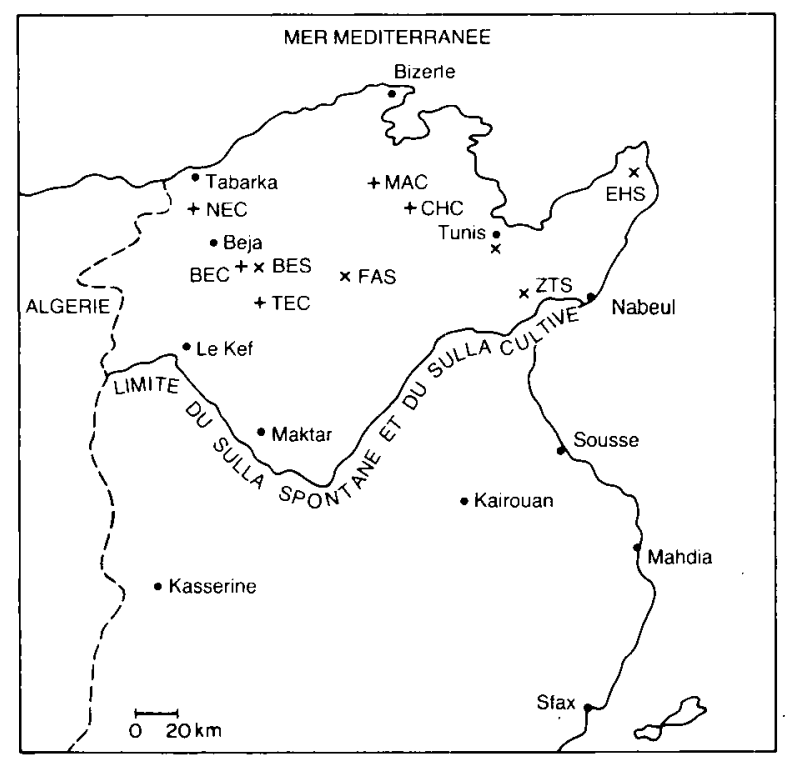

Fig. 1. Stations d'H. coronarium prospectées au nord de la Tunisie : + populations cultivées; $x$ populations spontanées. tropes est croisé avec un individu de la population EHS présentant un port érigé avec des plagiotropes peu développés. Les descendants $F 1$ obtenus sont autofécondés individuellement. On obtient ainsi des familles F2. ZE1 correspond à une de ces familles obtenues en utilisant comme \& au départ un individu ZTS, tandis que EZ1, comme point de départ une $\uparrow$ de la population EHS.

\section{Méthodes}

\section{Systèmes enzymatiques étudiés}

II s'agit de 10 systèmes appartenant aux classes d'enzymes suivantes:

- oxydoréductases : alcool déshydrogénase (ADH), malate déshydrogénase (MDH), isocitrate déshydrogénase (ICD), phosphogluconate déshydrogénase (6$P G D)$, peroxydases (PER);

- transférases : glutamate oxaloacétate transaminase (GOT), phosphoglucomutase (PGM);

- hydrolases : estérases (EST), phosphatases acides (PHO);

- isomérase : phosphogluco-isomérase (PGI).

Pour chaque système enzymatique et chaque population, la taille moyenne de l'échantillon est de 24 . Pour l'étude des ségrégations, la taille des échantillons varie de 14 à 32 .

\section{Protocole expérimental}

Nous avons adopté le protocole expérimental décrit par Sandmeier et al. (1981) et Trigui et al. (1986).

Extraction : sept enzymes (GOT, ADH, 6-PGD, PGI, $P G M, M D H, I C D)$ ont été étudiées sur des germinations étiolées broyées dans le tampon ascorbate de $\mathrm{Na}(8,3 \%, \mathrm{pH} 8,4)$ additionné de $16,7 \%$ de saccharose et de $0,03 \%$ de $\beta$-mercaptoéthanol. Extraites par du Tris- $\mathrm{HCl}(0,2 \mathrm{M}, \mathrm{pH} 8,5)$ additionné de $0,03 \%$ de $\beta$-mercaptoéthanol, les PER et les PHO ont été révélées sur des racines prélevées sur des plantes âgées de 3 mois; les EST ont été étudiées sur des graines sèches.

Electrophorèse : seules les estérases ont été séparées sur gel de polyacrylamide $(7,5 \%)$ par une électrophorèse monodimensionnelle verticale dans un tampon de migration tris-glycine $(\mathrm{pH} \mathrm{8,3)}$. La migration anodique dure trois heures sous un voltage constant de $200 \mathrm{~V}$. La séparation sur gel d'amidon des isoenzymes des neuf enzymes étudiées a été réalisée par une électrophorèse monodimentionnelle horizontale.

Ainsi les PER et les PHO ont été séparées sur gel d'amidon (14\%) avec un tampon de gel et de migration tris-maléique $(\mathrm{pH} 7,0)$ en utilisant un voltage constant de $300 \mathrm{~V}$; la durée de la migration est de $16 \mathrm{~h}$.

La séparation des isoenzymes des GOT et des $\mathrm{ADH}$ sur gel d'amidon (13\%) a été réalisée avec un tampon de gel tris-citrate $(\mathrm{pH} \mathrm{8,3)} \mathrm{et} \mathrm{un} \mathrm{tampon} \mathrm{de}$ migration lithium-borate $(\mathrm{pH} 8,3)$ sous un ampérage constant égal à $40 \mathrm{~mA}$. La durée de la migration est de $6 \mathrm{~h}$. Les isoenzymes de 6-PGD, PGM, PGI, MDH, et ICD ont été séparées sur gel d'amidon à $13 \%$ en utilisant un tampon de gel tris-citrate $\mathrm{pH} 8,3$ et un tampon de migration lithium-borate $(\mathrm{pH} 8,3)$ sous une puissance constante de $16 \mathrm{~W}$. Dans ce cas la migration dure $7 \mathrm{~h}$.

La composition des solutions de révélation est peu modifiée par rapport à celle décrite par Scandalios 
(1969) pour l'alcool déshydrogénase, Stuber et al. (1977) pour la phosphogluconate déshydrogénase. La méthode d'Uriel (1964) permet la révélation des estérases, celle de Pai et al. (1975) est utilisée pour révéler les phosphatases acides. La malate déshydrogénase, l'isocitrate déshydrogénase, la phosphoglucomutase et la phosphogluco-isomérase ont été révélées selon Cardy et al. (1980). Le mélange des substrats de la $P G \mid$ et de la $P G M$ permet de révéler simultanément sur le même gel les deux enzymes qui ont des distances de migration différentes. La méthode de révélation des peroxydases est tirée de Shaw et Prasad (1970), celle de la glutamate oxaloacétate transaminase de Goodman et al. (1980).

\section{Résultats}

L'ensemble des profils électrophorétiques issus de toutes les populations analysées, a permis de dresser pour chaque système enzymatique les zymogrammes correspondants.

Toutes les interprétations se sont inspirées des principes de l'analyse génétique développée par Pernès (1984). Dans ce cadre nous retenons pour chaque système enzymatique l'hypothèse explicative la plus simple.

\section{Alcool déshydrogénase}

Pour l'ADH deux profils ont été obtenus (Fig. 2) : un profil à une seule bande et un profil à 3 bandes situées dans une même zone de migration. Ce résultat est en faveur de l'hypothèse la plus simple à savoir une structure dimérique de I'ADH avec un contrôle du type monogénique à 2 allèles ayant des interactions. En effet, selon cette hypothèse, les individus hybrides sont à 3 bandes, ce qui montre l'existence d'interaction entre les produits des allèles.

\section{Malate déshydrogénase}

Pour la $\mathrm{MDH}$, deux profils ont été obtenus (Fig. 3). Ces deux zymogrammes peuvent s'expliquer par une hypothèse simple selon laquelle

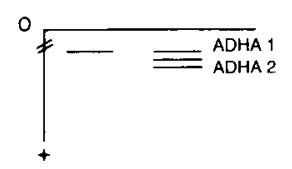

Fig. 2. Zymogrammes d'ADH.

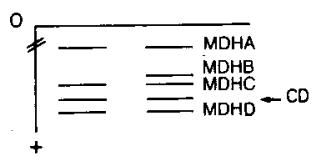

Fig. 3. Zymogrammes de MDH.
4 gènes au moins dont 3 monomorphes sont impliqués dans la synthèse de la MDH (locus $A$, locus $C$ et locus $D$ ). Le locus polymorphe B présente 2 allèles dont l'un est inactif. La bande notée $C D$ migrant à une distance intermédiaire entre les bandes $C$ et $D$ correspondrait, dans le cadre d'une structure dimérique de l'enzyme, à leur interaction.

\section{Isocitrate déshydrogénase}

Les profils de l'ICD présentés dans la figure 4 suggèrent l'hypothèse d'une structure dimérique contrôlée par un seul gène à 3 allèles avec des interactions possibles entre les allèles.

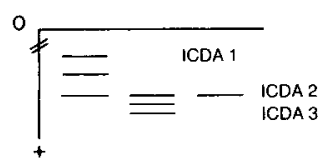

Fig. 4. Zymogrammes de ICD.

\section{Phosphogluconate déshydrogénase}

Dans la figure 5, nous représentons les différents électrophorégrammes de la 6-PGD de $H$. coronarium. En supposant une structure dimérique de cette enzyme, son contrôle génétique pourrait s'expliquer par une hypothèse impliquant 3 gènes : le gène PGDA avec 2 allèles dont l'un est amorphe, le gène PGDB ayant également 2 allèles qui sont actifs tous les deux et le gène PGDC monomorphe.

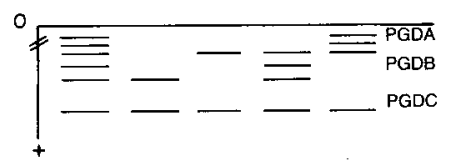

Fig. 5. Zymogrammes de 6-PGD.

L'existence d'interactions interlocus (cas des gènes PGDA et PGDB) et intralocus (cas du gène $P G D B$ ), est en accord avec l'hypothèse d'une structure dimérique de la 6-PGD chez $H$. coronarium.

\section{Peroxydases}

Les profils électrophorétiques observés pour les PER sont rassemblés dans la figure 6 . II en ressort que le contrôle de l'activité de ce système enzymatique serait dû à 5 gènes au moins :

- un gène pour les espèces cathodiques (PERA) avec deux allèles codominants. Les peroxydases contrôlées par ce gène seraient monomériques; 


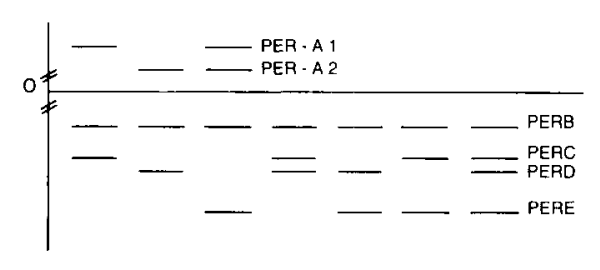

Fig. 6. Zymogrammes des PER.

- 3 gènes à deux allèles dont l'un est inactif (cas de PERC, PERD et PERE);

- un locus PERB monomorphe.

\section{Glutamate oxaloacétate transaminase}

Les zymogrammes obtenus pour cette transférase, sont présentés dans la figure 7. L'analyse de cette figure montre que l'activité de la GOT est gouvernée par au moins 5 gènes :

- trois locus présentant 2 allèles dont un inactif au niveau de chaque gène (cas des locus GOTA, GOTC et GOTD);

- un locus appelé GOTB possèdant 2 allèles codominants;

- un locus GOTE présenterait 3 allèles dont l'un est nul, dans ce cas la structure de la GOT serait monomérique.

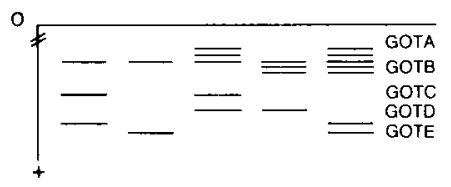

Fig. 7. Zymogrammes des GOT.

Cependant nous ne pouvons écarter l'hypothèse de 2 locus avec un système présenceabsence. Dans le cadre de cette deuxième hypothèse l'enzyme serait contrôlée par 6 locus au lieu de 5.

L'existence d'interactions entre les locus GOTA, GOTB d'une part et entre les allèles d'un même locus (GOTB) d'autre part, est en faveur d'une structure dimérique de cette enzyme.

\section{Phosphoglucomutase}

La figure 8 représente les profils enzymatiques obtenus pour cette enzyme. En formulant l'hypothèse d'une structure monomérique (les individus hybrides étant à 2 bandes) la PGM serait sous le

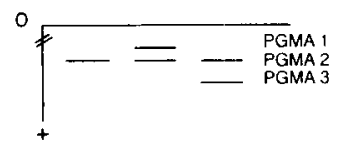

Fig. 8. Zymogrammes de PGM. contrôle d'un seul gène ayant 3 allèles codominants.

\section{Estérases}

L'analyse des différents profils permet de révéler l'existence de 13 bandes isoenzymatiques (Fig. 9). Le nombre de bandes par profil varie de 1 à 5 .

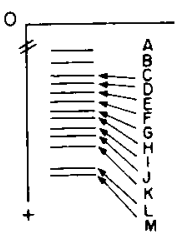

Fig. 9. Zymogrammes des EST.

Etant donné le nombre important de bandes et afin de faciliter l'analyse, nous avons tenu compte seulement de la présence ou de l'absence de bandes tout en supposant pour chacune d'entre elles un contrôle monogénique. Cette démarche simplificatrice face à des zymogrammes complexes a été utilisée chez d'autres espèces en particulier le seigle (céréale) et Secale vavilovii (Perez de la Vega et Allard, 1984). Ainsi 13 gènes au moins correspondant aux 13 bandes observées seraient impliqués dans le contrôle génétique des estérases.

\section{Phosphatases acides}

Les profils électrophorétiques sont schématisés dans la figure 10. En admettant l'hypothèse d'une structure monomérique, les PHO seraient codées vraisemblablement par 4 gènes : l'un monomorphe (PHOA), deux bialléliques (PHOC, $\mathrm{PHOD}$ ) avec un allèle nul pour chacun d'entre eux, et un dernier ayant 2 allèles codominants (PHOB).

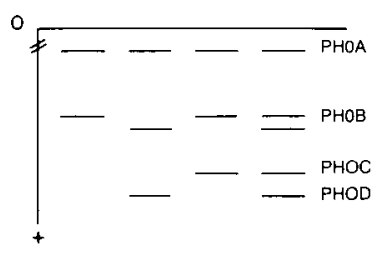

Fig. 10. Zymogrammes de PHO.

\section{Phosphogluco-isomérase}

Pour interpréter les profils observés pour cette isomérase (Fig. 11) nous avons émis l'hypothèse suivante : le contrôle génétique est du type monogénique avec 4 allèles présentant des interactions entre eux. L'existence de ces interactions suggère une structure dimérique de l'enzyme. 


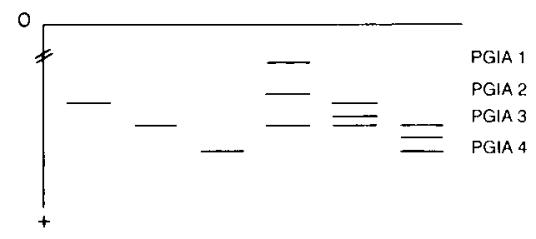

Fig. 11. Zymogrammes de PGI.

En conclusion, l'analyse des différents profils électrophorétiques obtenus pour les systèmes enzymatiques utilisés nous a permis d'émettre des hypothèses relatives à la structure et au déterminisme génétique de chacun d'entre eux.

\section{Analyse mendélienne}

Pour vérifier les différentes interprétations avancées, nous avons fait une étude mendélienne en considérant la ségrégation des bandes isoenzymatiques de descendants issus d'autofécondation d'un individu de la population spontanée $\mathrm{EI}$ Haouaria (EHS) noté E5 et d'un individu noté Z5 de la population spontanée de Djebel Zit (ZTS). Cette analyse ayant porté sur les systèmes GOT, $P G I$ et 6-PGD, a été réalisée sur des germinations des différents descendants obtenus par autofécondation des individus $E 5$ et $Z 5$. Les vérifications des hypothèses émises précédemment sont basées sur les phénotypes des descendants. Toutefois les génotypes des parents ne peuvent pas être connus étant donné que cette analyse utilise des germinations.

Notons que nous insisterons surtout sur les aspects qualitatifs des ségrégations, étant donné les effectifs limités des descendants.

Cas de la GOT Les profils enzymatiques des familles E5 et Z5 sont résumés dans la figure 12. Le polymorphisme est observé uniquement pour les locus GOTA, GOTD et GOTE. Les deux autres gènes se sont montrés monomorphes et les individus sont donc homozygotes pour ces gènes. Les ségrégations phénotypiques observées pour chacun des gènes polymorphes sont du type 3.1. (Tableau I) ce qui est en faveur de l'existence d'un gène à 2 allèles avec dominance absolue. Etant absent dans tous les profils, l'allèle GOTE 1 du locus GOTE n'a pas été considéré dans cette étude.
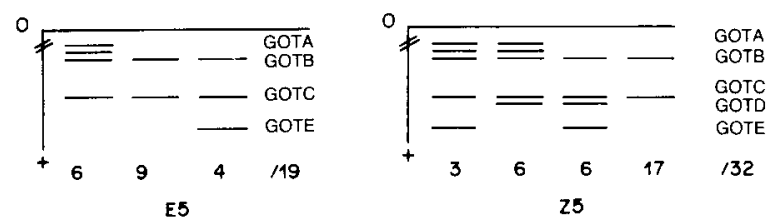

Fig. 12. Zymogrammes des GOT analysés à partir des autofécondations de $E_{5}$ et $Z_{5}$.
Tableau I. Analyse Mendelienne des GOT.

Tableau la. Ségrégation phénotypique pour chacun des locus GOTA et GOTE chez E5.

\begin{tabular}{lcccc}
\hline Phénotypes & A1 & A0 & E2 & E0 \\
& & & & \\
\hline Proportions & 1 & 3 & 1 & 3 \\
Effectifs observés & 6 & 13 & 4 & 15 \\
Effectifs théoriques & 4,75 & 14,25 & 4,75 & 14,25 \\
& & & & \\
$\chi^{2}$ & & 0,423 & & 0,149 \\
& & N.S. & & N.S. \\
\hline
\end{tabular}

N.S. : $\chi^{2}$ non significatif; $\chi^{2}$ th $=3,84$; seuil $5 \% 1$ ddl.

Tableau lb. Ségrégation phénotypique pour chacun des locus GOTA, GOTD et GOTE chez Z5.

\begin{tabular}{lrrrrrr}
\hline Phénotypes & A1 & A0 & D1 & D0 & E2 & EO \\
\hline Proportions & 1 & 3 & 1 & 3 & 1 & 3 \\
Effectifs observés & 9 & 23 & 12 & 20 & 9 & 23 \\
Effectifs théoriques & 8 & 24 & 8 & 24 & 8 & 24 \\
$\chi^{2}$ & 0,166 & 2,666 & 0,166 \\
& & N.S. & N.S. & N.S. \\
\hline
\end{tabular}

N.S. : $\chi^{2}$ non significatif; $\chi^{2}$ th $=3,84 ;$ seuil $5 \% 1$ ddl.

Cas de la PGI Seule la famille Z5 a présenté un polymorphisme pour cette enzyme (Fig. 13). La ségrégation observée pour les allèles PGIA 1 et PGIA 3 du type 1.2.1. (Tableau II), suggère un contrôle par un gène ayant 2 allèles codominants (PGIA 1 = PGIA 3). Le parent Z5 serait donc hétérozygote et de génotype PGIA 1 / PGIA 3.

Cas de la 6-PGD La figure 14 résume l'ensemble des profils obtenus chez les familles E5 et Z5.

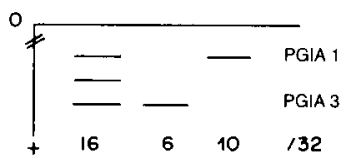

Fig. 13. Zymogrammes de PGI analysés à partir des autofécondations de $Z_{5}$.

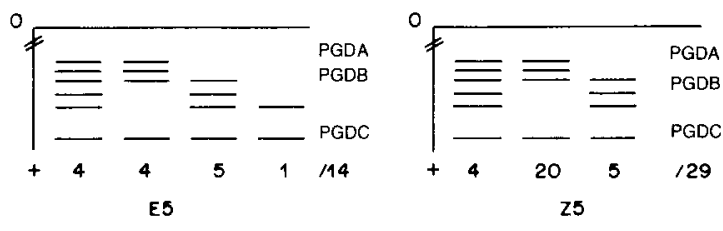

Fig. 14. Zymogrammes de 6-PGD analysés à partir des autofécondations de $E_{5}$ et $Z_{5}$. 
Tableau II. Analyse Mendelienne de PGI chez la famille Z5.

\begin{tabular}{|c|c|c|c|c|}
\hline Phénotypes & A1 & & $\mathrm{A} 1 \mathrm{A3}$ & A3 \\
\hline Proportions & 1 & & 2 & 1 \\
\hline Effectifs observés & 10 & & 16 & 6 \\
\hline Effectifs théoriques & 8 & & 16 & 8 \\
\hline$\chi^{2}$ & & 1 & & N.S. \\
\hline
\end{tabular}

N.S. : $\chi^{2}$ non significatif; $\chi^{2}$ th $=5,99 ;$ seuil $5 \%, 2$ ddl.

Concernant le gène PGDA, la ségrégation vraisemblablement du type 3.1. (Tableau IIla) permet de conclure à l'existence d'un locus à 2 allèles avec dominance absolue. Dans le cadre de cette hypothèse, les parents seraient hétérozygotes et de génotype PGDA 1 / PGDA 0 avec dominance de PGDA 1 sur PGDA 0.

Pour le gène PGDB (Tableau IIIb), le polymorphisme semble être également dû à 2 allèles codominants. Cette hypothèse est confirmée pour l'individu E5 dont la ségrégation est du type 1.2.1. Cependant la ségrégation de $Z 5$, qui ne semble pas être en accord avec cette interprétation, s'expliquerait par une sélection en faveur de

Tableau III. Analyse mendelienne de PGD. Tableau Illa. Ségrégation pour le locus PGDA.

\begin{tabular}{lcccl}
\hline & \multicolumn{2}{c}{ E5 } & \multicolumn{2}{c}{ Z5 } \\
Phénotypes & A1 & A0 & A1 & A0 \\
\hline Proportions & 3 & 1 & 3 & 1 \\
Effectifs observés & 8 & 6 & 24 & 5 \\
Effectifs théoriques & 10,5 & 3,5 & 21,75 & 7,25 \\
$\chi^{2}$ & 2,38 & N.S. & 0,930 & N.S. \\
\hline
\end{tabular}

N.S. : $\chi^{2}$ non significatif; $\chi^{2}$ th $=3,84$; seuil $5 \% 1$ ddl.

Tableau Illb. Ségrégation pour le locus PGDB.

\begin{tabular}{llllllll}
\hline & \multicolumn{3}{c}{ E5 } & \multicolumn{3}{l}{ Z5 } \\
Phénotypes & B1 & B1B2 & B2 & B1 & B1B2 B2 \\
\hline Proportions & 1 & 2 & 1 & 1 & 2 & 1 \\
Effectifs observés & 4 & 9 & 1 & 20 & 9 & 0 \\
Effectifs théoriques & 3,5 & 7 & 3,5 & 7,25 & 14,5 & 7,25 \\
$\chi^{2}$ & 2,43 & N.S. & 31,75 & $S$ \\
\hline$S: \chi^{2}$ significatif; N.S. : $\chi^{2}$ non significatif; $\chi^{2}$ th $=5,95$; seuil $5 \% 2$ ddl.
\end{tabular}

l'allèle PGDB 1. D'ailleurs la faible fréquence de l'allèle PGDB 2, obtenue chez l'ensemble des populations analysées, est en accord avec cette hypothèse.

\section{Conclusion et Discussion}

Dans cette étude, et grâce à l'analyse de la variabilité enzymatique observée dans différentes populations de $H$. coronarium, nous avons pu émettre des hypothèses pour expliquer aussi bien la structure que le contrôle génétique de dix systèmes enzymatiques. Le tableau IV regroupe l'essentiel des résultats.

Mise à part l'ADH, les données génétiques obtenues sont dans l'ensemble comparables à celles rapportées pour d'autres espèces dont certaines appartenant à la famille des Légumineuses.

Ainsi les résultats concernant l'ADH, quoique mettant en évidence la présence d'un seul gène, ont permis de confirmer la structure dimérique de l'enzyme, telle qu'elle a été montrée chez d'autres espèces. (Pour une revue voir Scandalios, 1969). Quoiqu'il en soit, l'hypothèse de deux gènes impliqués dans le contrôle de l'ADH chez $H$. coronarium n'est pas à exclure. Dans ce cas, le deuxième locus serait complètement inactif.

Le contrôle multigénique de la MDH a été également démontré chez plusieurs espèces dont le maïs (Cardy et al., 1980), le seigle (céréale) et Secale vavilovii (Perez de la Vega et Allard, 1984) et le mil (Trigui, 1984).

La structure dimérique et le contrôle monogénique de l'ICD ont été observés chez d'autres espèces, en particulier chez la lentille (Zamir et Ladizinsky, 1984), le caféier (Berthaud, 1984) et également le mil (Trigui et al., 1986) etc.

La structure dimérique de la 6-PGD a été signalée chez le blé (Hsam et al., 1982) et le caféier (Berthaud, 1984). Le contrôle polygénique a été montré chez le mil (Trigui et al., 1986), et Secale cereale (Salinas et Benito, 1983).

La structure monomérique et le déterminisme polygénique des $\mathrm{PER}$ avec des allèles nuls ont été retrouvés chez d'autres espèces (Edwin, 1974; Garcia et al., 1982; Perez de la Vega et Allard, 1984).

Chez $H$. coronarium, la GOT a présenté une structure dimérique et monomérique. Cependant chez plusieurs autres espèces, la GOT serait un dimère (Scandalios et al., 1975, Mahmoud et al., 1984). Le contrôle multigénique a été également signalé chez d'autres espèces (Zamir et Ladizinsky, 1984; Cardy et al., 1980). 
Tableau IV. Récapitulation des caractéristiques des différentes enzymes.

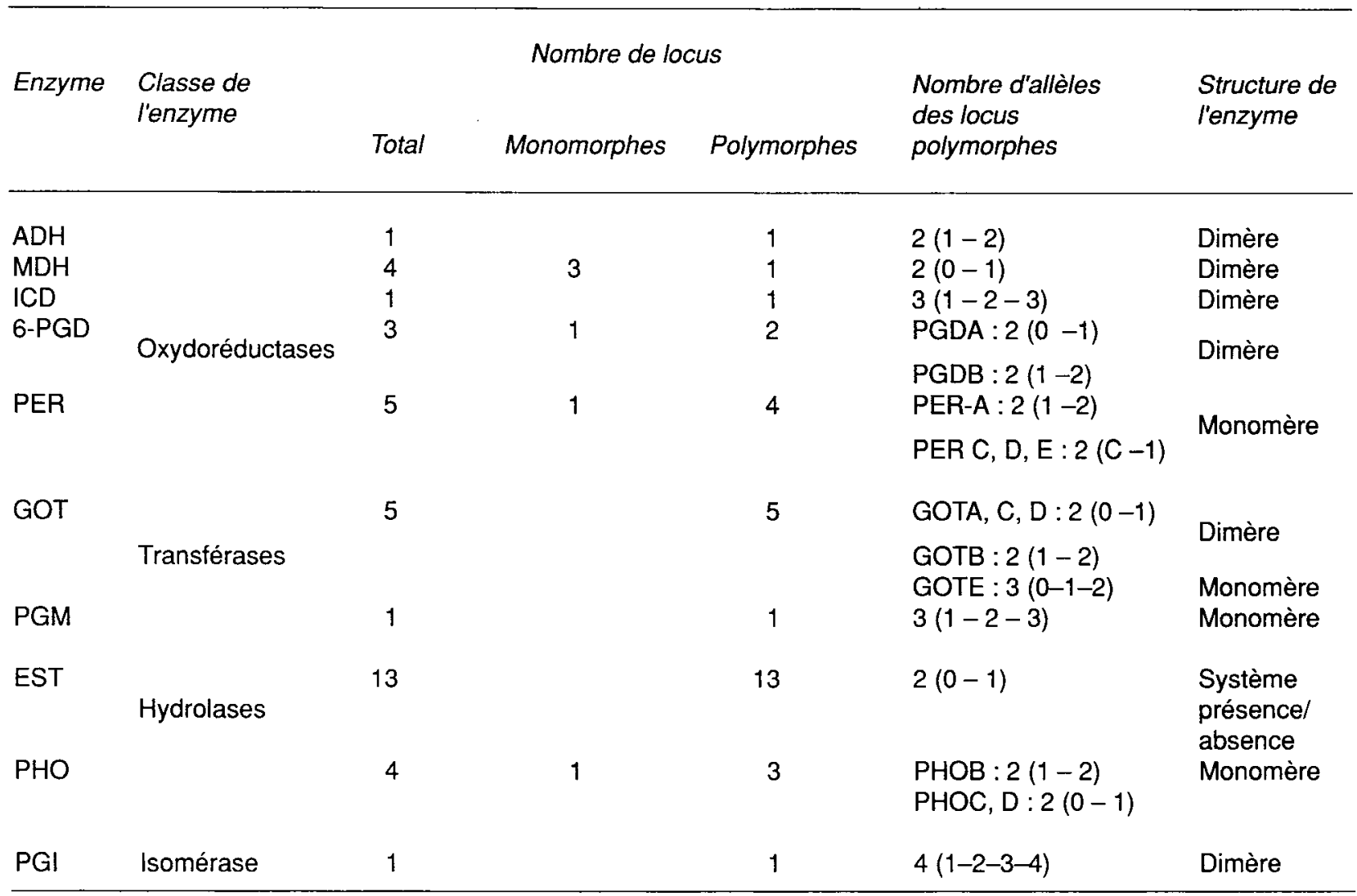

Concernant la PGM, des résultats analogues ont été observés chez l'orge (Brown et al., 1978), le mil (Le Blanc et Pernès, 1983), le seigle (céréale) et Secale vavilovii (Perez de la Vega et Allard, 1984).

Chez d'autres espèces, le même type de contrôle de la PHO est retrouvé (Jacobs et Schwind, 1974), cependant cette enzyme se comporte généralement comme un dimère (Salinas et al., 1981).

Pour la PGI, des résultats analogues ont été obtenus chez d'autres espèces végétales, en particulier la pomme de terre (Staub et al., 1984).

Par ailleurs, certaines de ces hypothèses ont pu être vérifiées grâce à la ségrégation des bandes isoenzymatiques dans la descendance de deux familles issues d'autofécondation (GOT, 6-PGD et PGl).

II est cependant important de noter que l'utilisation de parents dont on connaît les génotypes et l'analyse d'un échantillon plus important pourraient permettre de mieux élucider les hypothèses proposées.

Enfin, il faut souligner, à côté de l'intérêt que présentent ces données pour déterminer le contrôle et la structure des enzymes, qu'elles seront exploitées pour l'étude des populations.

\section{Remerciements}

La mise au point de cet article a profité des suggestions de Monsieur le Professeur Mounolou J.-C. de l'Université Paris-Sud (Orsay). Nous remercions Monsieur Sandmeier M. du GPDP du CNRS pour ses conseils et fructueuses discussions. L'ACCT, la FIS et le CNRS ont contribué matériellement à la réalisation de ce travail.

\section{Références}

Baatout H., Boussaid D., Combes D., Espagnac-H. \& Figier J. (1976) Contribution à l'étude du genre Hedysarum en Tunisie. Bull. Soc. Sc. Nat. Tunisie, 11, 8795

Ballatore J.P. (1972) La produzione foraggera negli ambianti semiaridi, conparticolare refrimento alla Sicilia. L'Inf. Agrario. 27, 7433-7464

Berthaud J. (1984) Les ressources génétiques pour l'amélioration des caféiers africains diploïdes. Evaluation de la richesse génétique des populations sylvestres et de ses mécanismes organisateurs. Conséquences pour l'application. Thèse doctorat d'Etat, Univ. Paris-Sud (Orsay)

Brown A.H.D., Nevo E., Zohary D. \& Dagan O. (1978) Genetic variation in naturel populations of wild Barley (Hordeum spontaneum). Genetica (The Hague) 49, 97-108

Cardy B.H., Stuber C.W. \& Goodman M.M. (1980) 
Techniques for starch gel electrophoresis of enzymes form maize (Zea mays L.). Inst. Statistics mimeograph. North. Carolina State University, Raleigh. North Carolina, $n^{\circ} 1317$

Cenni B., Jannella G. \& Colombani N. (1968) Chemical composition, digestibility and nutritive value of sulla (Hedysarum coronarium L.) high produced in Voltera district. Ann. Fac. Med. Vet. Univ. Pisa 20, 155-168

Chatti W.S. (1987) Analyse de la diversité génétique basée sur les caractères morphologiques et le polymorphisme enzymatique des espèces Hedysarum coronarium L. et Hedysarum carnosum Desf. Relations phylogéniques avec le complexe Hedysarum spinosissimum L.s.l. Thèse $3^{\mathrm{e}}$ cycle, Faculté des Sciences, Tunis

Chriki A. (1986) L'hérédité des anthocyanes florales chez Hedysarum coronarium L., H. carnosum Desf. et H. capitatum Desf. Asch et Gr. Thèse Doctorat d'Etat, Univ. Pau et des Pays de l'Adour

Chriki A., Combes D. \& Marrakchi M. (1984) Etude de la compétition pollinique chez le sulla (Hedysarum coronarium L.). Agronomie, 4, 155-159

Combes D., Espagnac H. \& Figier J. (1975) Etude de populations naturelles d'Hedysarum coronarium $\mathrm{L}$. du nord de la Tunisie. Bull. Soc. His. Nat. Afr. Nord. 66, 107-122

Edwin H.L. (1974) Substrate specificity of plant peroxydase isozymes. In : II. Isozymes : Physiological function. Edited by Market C.L. Academic Press, New York, pp. 837-849

Figier J., Espagnac H. \& Combes D. (1977) Etude de populations naturelles d'Hedysarum coronarium $\mathrm{L}$. du nord de la Tunisie : comparaison en culture expérimentale. Bull. Soc. Bot. Fr., 124, 529-541

Figier J. (1982) Etude de la variabilité et du déterminisme de la morphologie de l' Hedysarum coronarium L. en Tunisie. Implication concernant l'amélioration de cette espèce fourragère dans ce pays. Thèse doctorat Etat, Univ. Paris-Sud (Orsay)

Garcia P., Perez de la Vega M. \& Benito C. (1982) The inheritance of rye seed peroxydases. Theor. Appl. Genet., 61, 341-351

Goodman M.M., Stuber C.W., Newton K. \& Weissinger H.H. (1980) Genetic control of $\mathrm{MDH}$ isozymes in maize. Genetics, 94, 153-168

Hsam S.L.K., Zeller F.J. \& Hubber W. (1982) Genetic control of 6-PGD isozymes in cultivated wheat and rye. Theor. Appl. Genet., 62, 317-320

Jacobs M. \& Schwind F. (1974) Biochemical genetics of Arabidopsis phosphatases : polymorphism, tissue expression and genetics of $\mathrm{AP}_{1}, \mathrm{AP}_{2}$ and $\mathrm{AP}_{3}$ loci. In : $I V$ : Isozymes : Plant and evolution. C.L. Market, ed. Academic Press, New York, pp. 349-369

Le Blanc J.M. \& Pernès J. (1983) Enzyme polymorphism of Pennisetum americanum in the Ivory Coast. Japan. J. Genet. 58, 12t-131

Le Houerou H.N. (1965) Les cultures fourragères en Tunisie. Documents techniques INRA, Tunisie 13

Mahmoud S.H., Gatehouse J.A. \& Boulter D. (1984) Inheritance and mating of isozymes in pea (Pisum sativum L.) Theor. Appl. Genet. 68, 559-566
Maymome B., Tibero M. \& Mazzioti di Celso P. (1951) Chemical composition and feed value of Hedysarum coronarium. Conf. for Improv. of past and fodder product. in the medit. area, Rome, pp. 3-19

Pai C., Endo T. \& Oka H.I. (1975) Genetic analysis for acid phosphatases isozymes in Oryza perennis and Oryza sativa. Can J. Genet. Cytol., 17, 637-650

Perez de la Vega M. \& Allard R.W. (1984) Mating system and genetic polymorphism in populations of Secale cereale and S. vavilovii, Can. J. Genet. Cytol. 26, 308-317

Pernès J. (1984) Gestion des ressources génétiques des plantes. Tome II : Manuel. (J. Pernès et al., ed.) A.C.C.T. Paris

Salinas J. \& Benito C. (1983) Chromosomal location of genes controlling 6-PGD, G6-PD and GDH isozymes in cultivated rye. Euphytica 32, 783-790

Salinas J., Perez de la Vega M. \& Benito C. (1981) The chromosomal location of phosphatase isozymes of the wheat endosperm. Experimentia 37, 557-558

Sandmeier M., Beninga M. \& Pernès J. (1981) Analyse des relations entre formes spontanées et cultivées chez le mil à chandelles. III : Etude de l'hérédité des estérases et des peroxydases anodiques. Agronomie 1, 487-494

Scandalios J.G. (1969) Genetic control of multiple molecular forms of enzymes in plants. A Review. Biochem. Genet. 3, 37-79

Scandalios J.G., Sorenson J.G. \& Ott L.A. (1975) Genetic control and intracellular localisation of Glutamate oxaloacetate transaminase in maize. Biochem. Genet. 13, 759-763

Shaw C.R. \& Prasad R. (1970) Starch gel electrophoresis of enzymes: A compilation of recipes. Biochem. Genet. 4, 297-320

Staub J.E., Kuhns L.J., Grun P. \& May N. (1984) Genetic bases of isozyme variation for alkaline phosphatase and glucose phosphate isomerase in Solanum. Theor. Appl. Genet. 67, 505-513

Stuber C.W., Goodman M.M. \& Johson F.M. (1977) Genetic control and racial variation of $B$ - glucosidase isozymes in maize (Zea mays) Biochem. Genet 15 , 383-394

Trigui N. (1984) La variabilité génétique des mils (Pennisetum typhoides Bur, Stapf et Hubb) de Tunisie. Etude biométrique et analyse du polymorphisme enzymatique. Thèse $3^{\mathrm{e}}$ cycle. Université Paris-Sud (Orsay)

Trigui N., Sandmeier M., Salanoubat, M. \& Pernès J. (1986) Utilisation des données enzymatiques et morphologiques pour l'étude des populations et de la domestication des plantes: I. Séparation et identification génétique d'isozymes chez le Mil (Pennisetum thyphoïdes Bur. Stapf. et Hubb). Agronomie 6 (9), 779 788

Uriel J. (1964) The caracterization reactions of the protein constituents following electrophoresis or immunoelectrophoresis in agar. In : Immuno-electrophoretic analysis. (Grabar et Burtin, ed. Elsevier Amsterdam, London, New York, pp. 30-57

Zamir D. \& Ladizinsky G. (1984) Genetics of allozymes variants and linkage group in Lentil. Euphytica 33, 329-336 